\title{
Single Shot Switch Performance on the Cannon Caliber Electromagnetic Gun Program
}

\author{
T.J. Hotz, C.E. Penney, J.R. Kitzmiller, and M.D. Werst \\ The University of Texas at Austin, Center for Electromechanics, Austin, Texas
}

\begin{abstract}
The Cannon Caliber Electromagnetic Gun (CCEMG) program at The University of Texas at Austin Center for Electromechanics incorporates two solid state switch modules. The $95 \mathrm{MW}$ rectifier/inverter bridge (RIB) generates the field inside of the compulsator (CPA) and the $825 \mathrm{kA}$ gun switch module (GSM) is the main closing switch between the CPA and the launcher. Since commissioning the CCEMG system, nearly 400 discharges have been performed using these switches. These tests produced a significant amount of information which will be very useful for the design of future solid state switching systems on other electromagnetic launch programs. This paper will present the basic design, testing and lessons learned from the commissioning of the CCEMG system switches.
\end{abstract}

\section{INTRODUCTION}

New electromagnetic gun systems being designed at The University of Texas at Austin Center for Electromechanics (UT-CEM) use switch technology developed during the CCEMG program. For this program, a four-pole air core compulsator was used to drive a $2.25 \mathrm{~m}$ augmented railgun capable of rapid firing 15 projectiles at a velocity of 1,850 $\mathrm{m} / \mathrm{s}$. The program was completed in Fall 1996 having performed over 400 tests including six single shot tests and demonstration of multishot dry fire capability [1]. During the CCEMG program, UT-CEM gained much knowledge in three different areas: compulsators, railguns, and high voltage high current switches. This paper will concentrate on the problems encountered and lessons learned from the switching systems of the CCEMG program.

The CCEMG system incorporates five different switch designs, each with unique operating requirements [2],[3]. An overall system schematic is shown in Fig. 1. The five include the field initiation module (FIM), rectifier/inverter bridge (RIB), gun switch module (GSM), explosive opening switch

Manuscript received May 1, 1998.

T.J. Hotz may be contacted at 512-471-4496, fax 512-471-0781, t.hotz@mail.utexas.edu

This research was supported by U.S. Army ARDEC and the U.S. Marine Corps under contract no. DAAA21-92-C-0062. Funding was provided by

The University of Texas.
(EOS) and a muzzle arc suppression switch. Only two of the switches will be covered in the paper, the RIB and GSM. The EOS and FIM histories have been well established at UTCEM ([3][4]) and are not addressed in this paper. Additionally, the muzzle arc suppression switch was designed but never built or tested and therefore will not be covered in this paper.

The RIB is a single phase full wave bridge responsible for field coil excitation and regenerative field coil dump. The four legs of the bridge each consist of six thyristors arranged in series/parallel configuration. Once the FIM has been discharged into the field coil, the RIB starts rectification until the desired level of excitation for particular test is achieved. The level is predetermined from simulation and entered as an input parameter to the main control system. The switch was designed with a goal of a maximum of $25 \mathrm{kA}$ in the field coil; however, $30.5 \mathrm{kA}$ was achieved on CCEMG run \#116. The other function of the RIB is inversion. Inversion is necessary to meet program goals of a 15 shot burst at $5 \mathrm{~Hz}$. Since a discharge only uses some of the energy stored in the field coil, the remaining energy can be used to help accelerate the rotor between salvos. Thus, after a discharge, the RIB is gated as an inverter and converts the stored electrical energy into kinetic energy.

The other switching system discussed in this paper is the GSM. The GSM consists of 40 parallel $4.5 \mathrm{kV}$ thyristors designed to deliver $825 \mathrm{kA}$ in pulsed duty mode. The GSM provided the path to deliver the current from the compulsator to the railgun during a discharge. The GSM was designed, built and tested as a snubberless system [2]. A normal discharge would last approximately $2.5 \mathrm{~ms}$ and is considered soft duty for these thyristors. Preliminary analysis determined that the $4.5 \mathrm{kV}$ limit would not be exceeded during normal operation of the CCEMG system even during turn-off of the devices.

\section{RECTIFIER/INVERTER BRIDGE}

\section{Mechanical Packaging}

The complete RIB is packaged in one enclosure. The single unit design allowed for the smallest volume and the best 


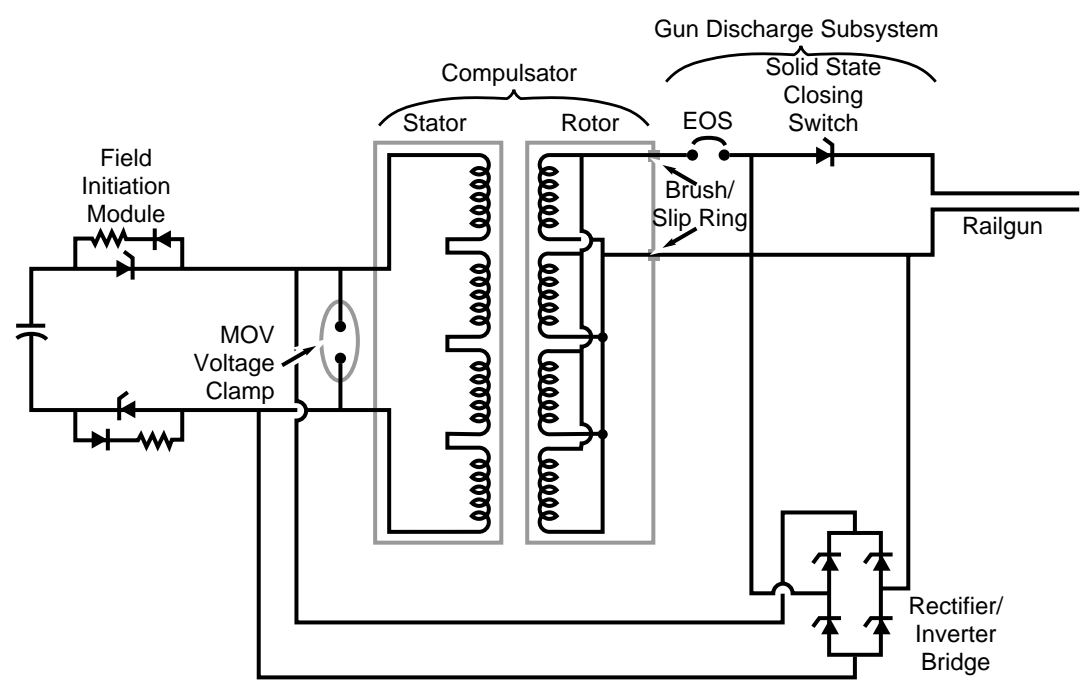

3010.0099

Fig. 1. Overall CCEMG system schematic.

matching of impedance between the parallel current paths. The R/I bridge contains four identical quadrants of six thyristors each. The six thyristors in a quadrant have an RC snubber circuit located above the thyristors. The ac input power is fed through flexible hexapolar cable into the center nodes of the thyristor stacks. The rectified dc output leaves from the top of the bridge assembly and returns from the field coil on the bottom. The bridge layout is shown in Fig. 2 and an assembly photo is shown in Fig. 3 .

The thyristor selected for the bridge is a custom version of the Powerex TD20, rated at 3,800 V. The Powerex TD20 was selected for performance and packaging reasons. This unit was available in a thin ceramic case, which reduced the over all stack height. The bridge is assembled in two identical halves. Each half is pressed together with a gang clamping system, meaning the preload is maintained using spring washers. Each half uses a pair of titanium flanges that have three self-aligning tilt pads held together with a single steel rod.

\section{Gate Drive}

One of the more critical areas of design for power electronic circuitry is proper device gating. Proper gating techniques are very important for reliably turning the thyristor on as well as for longevity of the device. Some of the earliest design work done on the CCEMG power electronics was to investigate what type of gate drive circuit would be optimal for this system [2]. This early study found the best method of gate drive for this system was a hard drive gate pulse with a $50 \mu \mathrm{s}$ back porch for the moderate to high di/dt and dv/dt requirements.

The basic gate drive circuit used for both the bridge and GSM is shown in Fig 4. For the hard gate drive pulse, a $60 \mathrm{~V}$, $3.5 \mathrm{~A}$ is generated at the output of the pulse transformer. A single pulse transformer, with eight secondary turns, gates each bridge quadrant, ensuring that all thyristors are turned on at

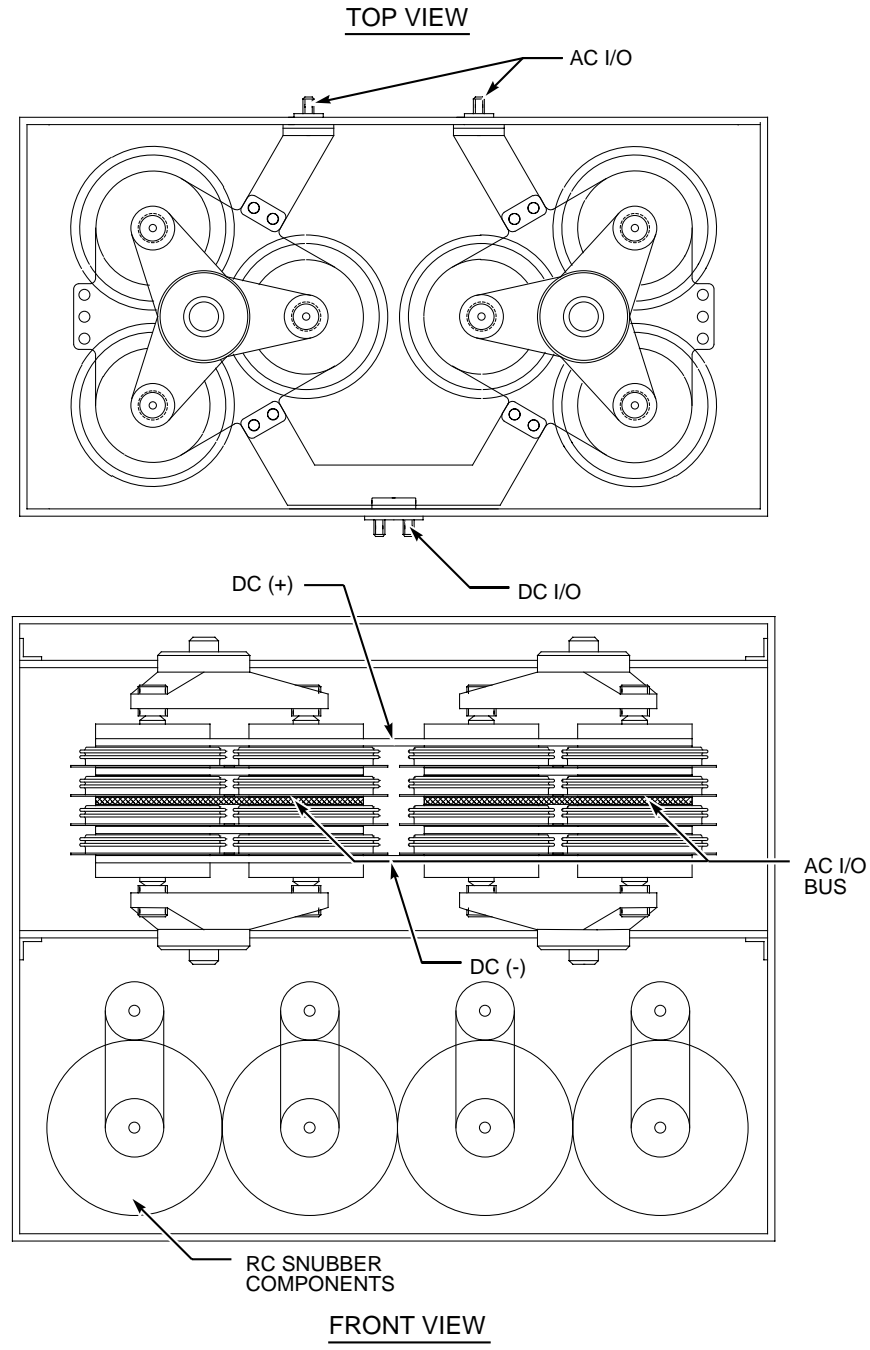

3010.0106

Fig. 2. Mechanical layout of RIB, top and front views. 
the same time. The IGBT has strict gating requirements much like a thyristor. To satisfy these requirements, a complementary FET pair was used. The gate drive circuits have turned the thyristor on with $100 \%$ reliability as long as there was a sufficient forward drop across the device (at least $40 \mathrm{~V}$ ). The timing of the gate pulses with respect to actual CPA voltage during a run is shown in Fig. 5. (Note that the gate pulses shown are the output of the pulse transformer and are optically isolated therefore not the actual gate pulse used to drive the thyristor).

\section{Current Monitors}

To monitor the actual current distribution between thyristors during operation, Rogowski coils are used to instrument each thyristor. The 24 Rogowski coil signals are conditioned and monitored by the bridge current sharing monitor (BCM). Since the bridge operates in quadrants, all thyristors in a quadrant are gated at the same time. Likewise, the BCM handles the 24 Rogowski signals as four groups of six signals each. After integration of the raw Rogowski signal, the average of each six channel quadrant is formed. This establishes a quadrant average value for each of the four quadrants. The minimum acceptable sharing threshold is then added and subtracted from each average signal. This process yields a range against which each of the 24 individual integrated Rogowski signals is compared. Any signal from an individual Rogowski falling outside of its associated range will generate an error condition.

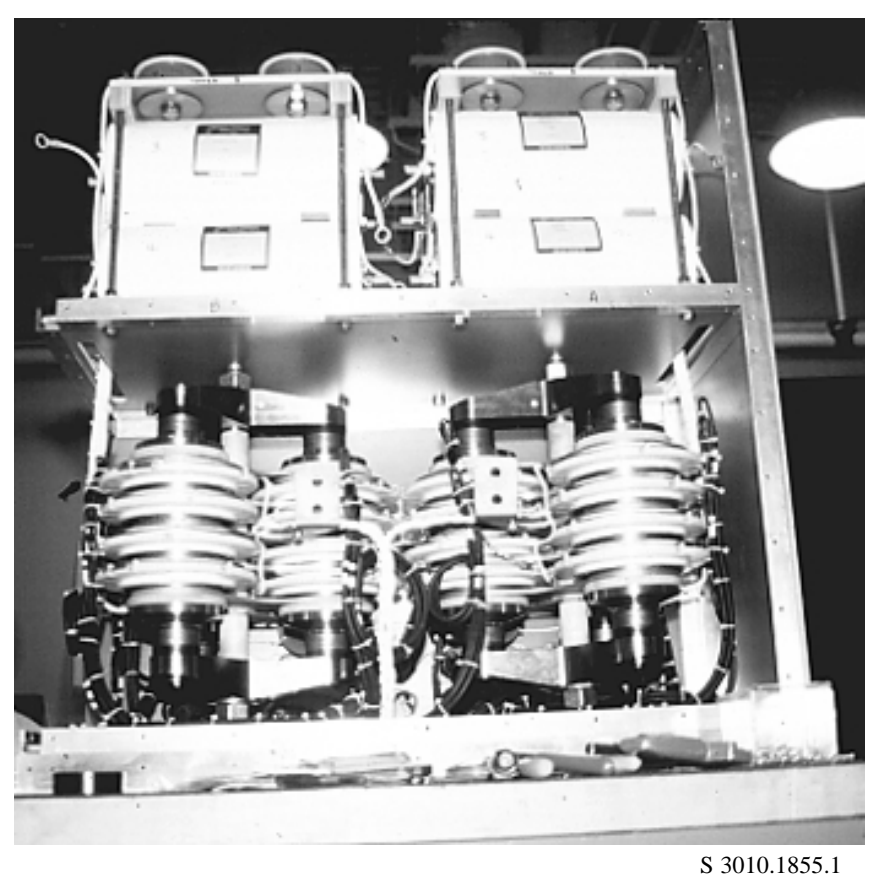

Fig. 3. Assembly of RIB.
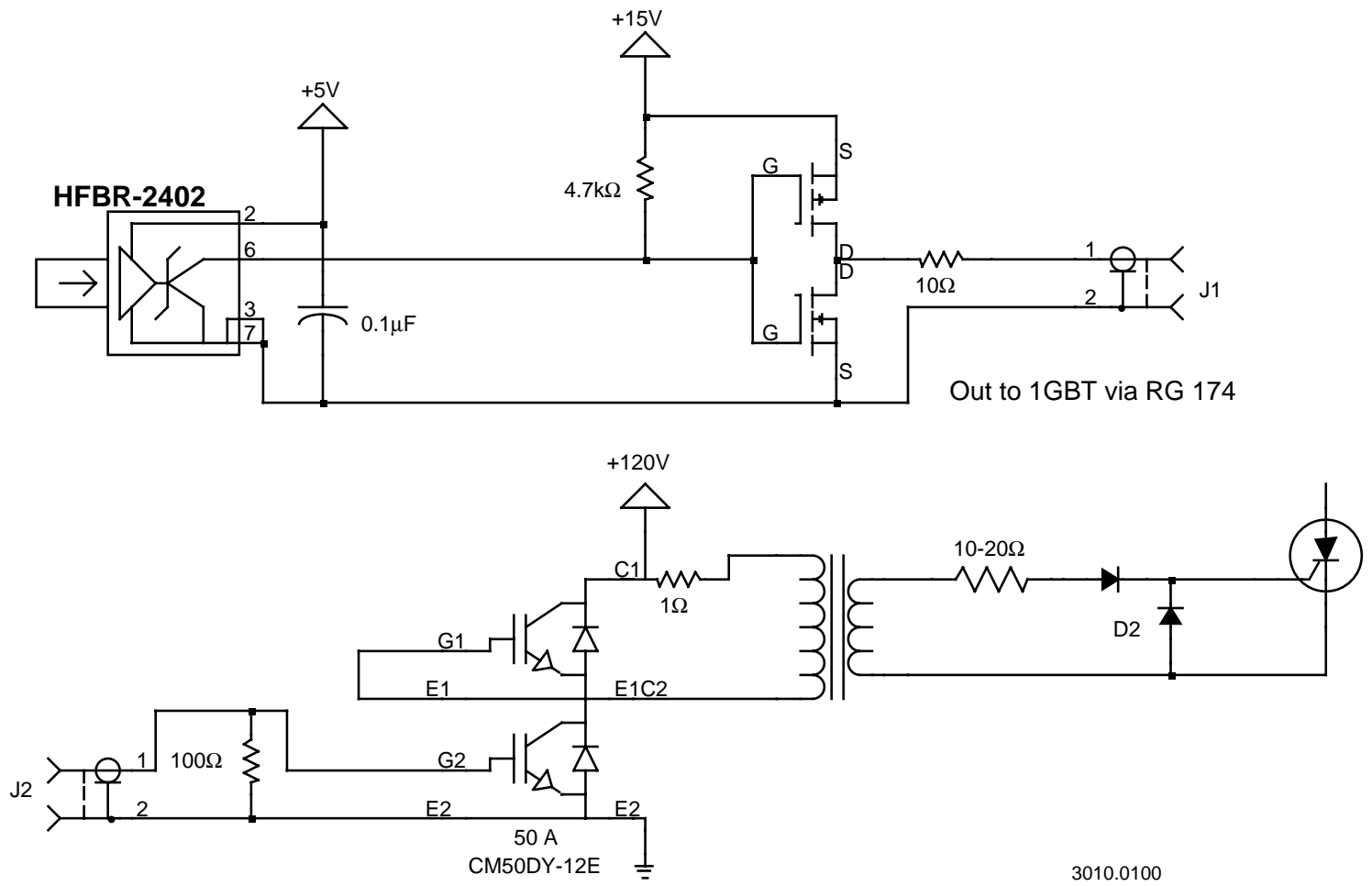

Fig. 4. Schematic of gate drive circuit. 


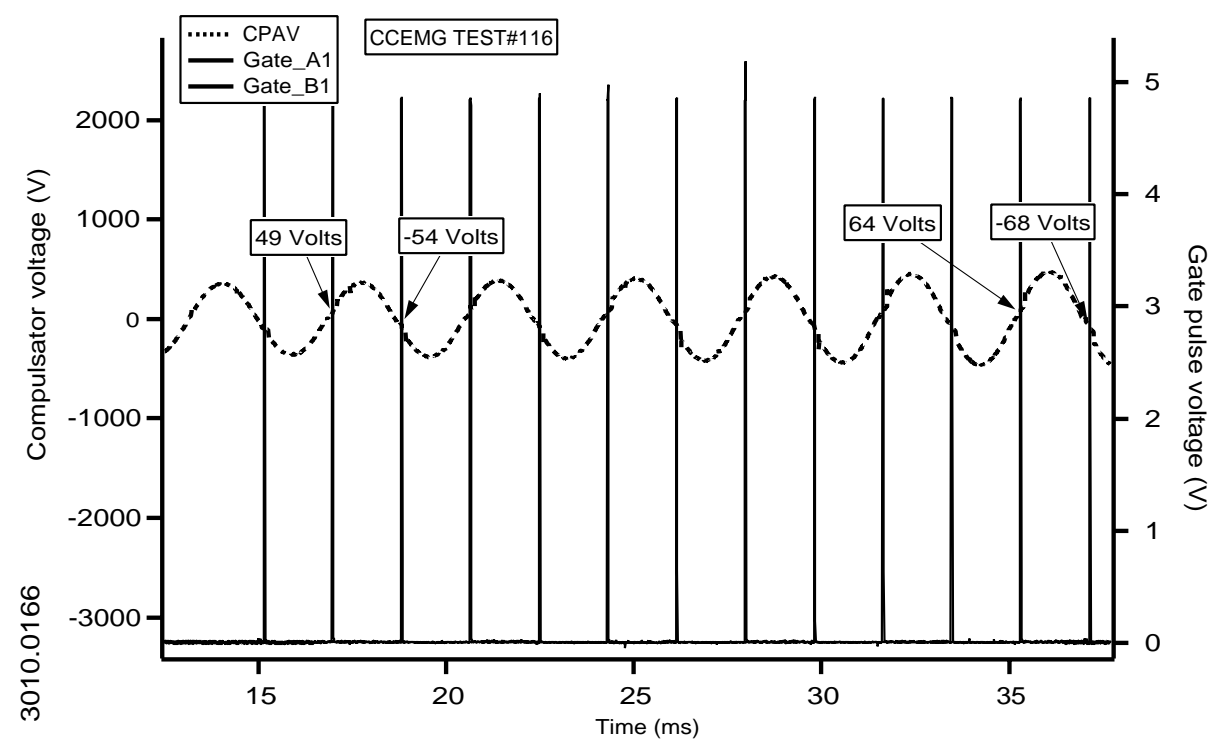

Fig. 5. Gate pulses and compulsator voltage.

In addition to average current, positive and negative current flow is indicated for each channel using a similar ranging technique. The positive and negative levels are fixed conduction thresholds for each of the forward and reverse directions. All three of the resultant signals (high, low, and negative current) are latched and displayed by quadrant on an array of LEDs.

The BCM suffered from a couple of minor problems. The CCEMG system was able to modify or work around these flaws. First, the BCM was designed to be an active control component, comparing current levels at all times. The problem with this system is that comparing current levels during turn on and off of the thyristors often produced errors indicating that the system is out of balance. It was learned that the current sharing could be compared about $100 \mu$ after gating the thyristors. Thyristor turn off produced no error in the CCEMG system; however, the potential for nonuniform sharing does exist. The solution used on the CCEMG system involved not using the BCM signals during the interval from thyristor gating to $100 \mu$ s after gating.

The second problem with the BCM involved the comparison levels. The BCM collects six signals, integrates them, sums them, and then divides by six to form the average signal. Adding a set value and subtracting a set value then forms a range of operation. At this point, each thyristor signal is compared to this range. The $\mathrm{BCM}$ as described can work quite well at any given current level. The wide operating range of the bridge rectifier causes the problem. A different approach would be to use a percentage of the current level to determine the size of the window instead of an absolute number.

The third problem with the operation of the BCM was noise. Two issues contributed to noise being a problem in the $\mathrm{BCM}$ system. One was small signal levels from the Rogowski coils at low current levels. The solution to low signal levels is a more sensitive Rogowski coil coupled with higher amplifier limits. The other problem on the CCEMG was a physical packaging problem. The CCEMG system was designed under aggressive size/weight requirements that resulted in a very tightly packaged bridge rectifier. The physical layout of the bridge has opposite polarity thyristor sets in the same stackup. The close proximity caused opposite polarity rectifying signals to be induced into the BCM system during large di/dt events. The point of entry of the erroneous signals is twofold: coupling at the Rogowski coil itself, and in the twisted shield pair wiring inside the bridge enclosure. Reducing the coupling proved to be a difficult task, in the given bridge enclosure. Shielding of the Rogowski coils was achieved by wrapping the coils with copper foil and attaching the copper shielding to ground. In testing after shielding, signal coupling was reduced to a reasonable level; however, it was not eliminated.

\section{Gun Switch Module}

Early testing during the CCEMG program called for the system to reach program goals for gun current and projectile velocity on a single shot test. The goal called for a discharge generating a gun current of $825 \mathrm{kA}$ and a muzzle velocity of $1,850 \mathrm{~m} / \mathrm{s}$ using a $185 \mathrm{~g}$ projectile. Once single shot testing began, it only took six shots to reach this goal. For all of the discharges, both dry and live fire, leading up to the sixth single shot gun shot the GSM performed flawlessly. However, on shot \#6 one of the 40 parallel thyristors broke down causing a short across the GSM. Although damage was minimal, the CCEMG team took a good hard look at the cause of the fault and subsequent design changes. 
In order to better understand the reasons for the fault, a thorough understanding of the entire system sequencing is needed. As mentioned earlier, the discharge sequence begins with a capacitive discharge of the FIM into the field coil. Once the FIM has completed its dump and the desired threshold is verified, the control system initiates the rectification mode of the RIB. The RIB ramps the field coil current up to the desired value entered into the control system prior to system operation. Once the desired field coil current level has been reached, the control system switches the RIB to freewheel mode and waits for two freewheel cycles before the GSM can be gated. After two freewheel cycles, the 40 thyristors of the GSM are gated simultaneously and the current is transferred from the compulsator to the railgun. For safe operation and maximum efficiency, the GSM is gated at the compulsator voltage reaches approximately $100 \mathrm{~V}$ in the forward direction. The gun current pulse lasts for approximately $2.5 \mathrm{~ms}$ and the thyristors turn off as the current in the GSM goes through zero.

Snubberless operation of the GSM would allow the program to meet weight and volume goals of the switching systems. Therefore, a high priority was put on making the snubberless design work. In many applications using thyristors, a snubber is used to lessen the voltage contribution from a fast turn off of the device. The voltage generated directly correlated to the induction in the circuit and the change in current as current passes through zero. This can be seen as:

$$
\mathrm{V}=\mathrm{L} \mathrm{di} / \mathrm{dt}
$$

Early analysis calculated the sum of the compulsator voltage at turn off and the $\mathrm{L} \mathrm{di} / \mathrm{dt}$ contribution would generate at peak voltage of $3,750 \mathrm{~V}$, resulting in a $20 \%$ margin of safety for the design using 40 thyristors in parallel [2]. The di/dt at turn off was calculated by simulation but had to be verified experimentally. The GSM was built and an iron core compulsator was used to test switch operation. The test sequence worked the current up in conservative steps with a watchful eye on turn off characteristics. Two tests were performed at a peak current of 833 and $847 \mathrm{kA}$ and the di/dt on these two test was measured at 457 and $444 \mathrm{MA} / \mathrm{s}$, respectively. Applying the impedance and di/dt measured on this test to the CCEMG system verified the $20 \%$ margin of safety for the switch.

With so much consideration and experimental verification given the design of the GSM, why was there a breakdown on shot \#6? Post analysis of the data from tests \#5 and 6 and subsequent analysis of the circuit point to potential weaknesses in the system. First of all, the circuit inductance was being measured experimentally on these gun shots to be about $1 \mu \mathrm{H}$ higher than the simulation yielded. Several voltage measurements were being taken during these tests to pin down the extra inductance. Additionally, the di/dt went unchecked during these tests and turns out to be $30 \%$ greater than anticipated. These factors led to a greater than anticipated turn off voltage contribution which is believed to have approached the rating of the thyristors. The peak voltage was measured at only $-3,394 \mathrm{~V}$ (Fig. 6), but the sample rate of the oscilloscope was insufficient to record the true peak. So, the circuit model was simulated to calculate the $\mathrm{L} \mathrm{di/dt} \mathrm{contribution} \mathrm{and} \mathrm{result-}$ ed in a $2,724 \mathrm{~V}$ spike generated at turn off using the new circuit inductance and $605 \mathrm{MA} / \mathrm{s} \mathrm{di} / \mathrm{dt}$ measured on shot \#5 (Fig. 7). The compulsator voltage at turn off was measured to be $1,924 \mathrm{~V}$. Because of circuit characteristics, the compulsator voltage was at its negative peak at turn off thus adding to the problem. Summing the two numbers would result in a voltage spike of 4,648 V which exceeds the 4,500 V rating of the GSM.

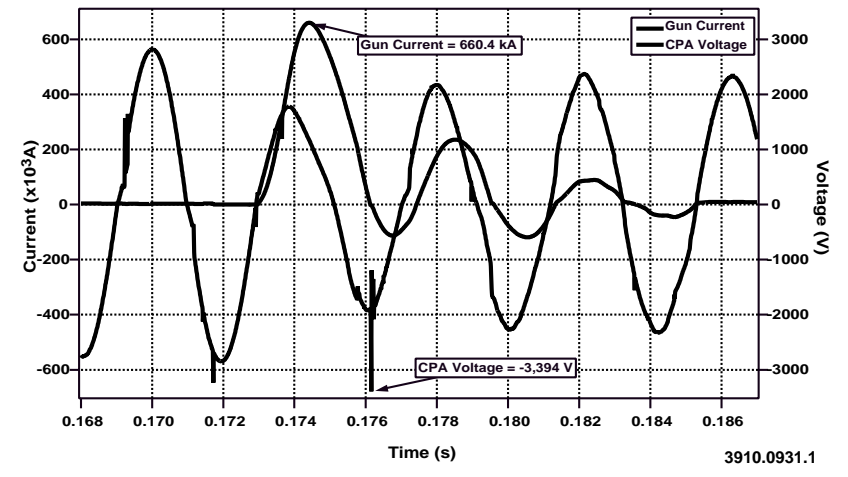

Fig. 6. Discharge data from CCEMG shot \#6.

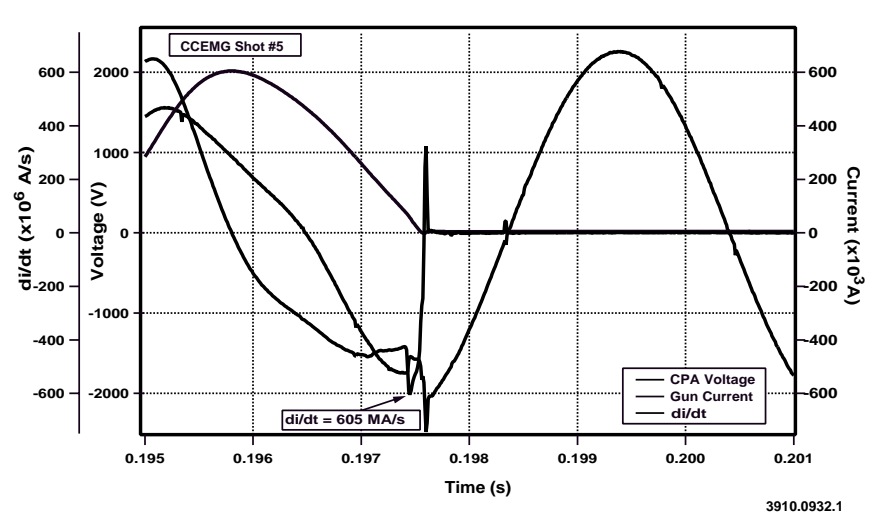

Fig. 7. Discharge data from CCEMG shot \#5. 
This new information meant that a snubber had to be added to the switch to lower the turn off contribution during high current tests. Additional information from the thyristor manufacturer indicated that the 4,500 $\mathrm{V}$ rating of the device had little or no safety margin. Consequently, a simple RC-snubber was designed and built. Prior to installation, lower current testing would be performed to experimentally verify the turn off contribution at a high sampling rate with new recording devices. By the end of the program the snubber had never been connected to the GSM because the system was never operated at a level which would approach the voltage rating of the devices.

\section{CONCLUSIONS}

Although the final program goals for the CCEMG program were not realized, UT-CEM gained invaluable knowledge about solid state switching systems on high voltage, high current applications. First, if Rogowskis are to be used for fault analysis and current sharing comparisons, then the different legs of the bridge should be package with the maximum possible distance between them to prevent flux coupling during turn on and turn off. Additionally, Rogowski design should include a means for shielding the coil and take careful consideration of signal to noise ratios for maximum effectiveness. Current monitors should ignore the sharing properties of the thyristors during the turn on period. The usefulness of current monitors at low current levels should be carefully considered.

Several important lessons were learned from the GSM as well. Unless advancements are made in thyristor technology, the larger switching systems of the future will require snubbers to prevent breakdowns. Additionally, all impedances should be well characterized both numerically and experi- mentally. Testing of components for future systems should go through extensive destructive testing to verify manufacturer's claims of device ratings. Additionally, circuit parameters should be closely monitored and designed in order to prevent peak system voltages from coinciding with the turn off time of the thyristors.

\section{ACKNOWLEDGMENTS}

The authors would like to acknowledge the numerous people from U.S. Army ARDEC, U.S. Marine Corps, UDLP, and UT-CEM who contributed to making the CCEMG system a reality. Funding for this effort has been provided by the University of Texas at Austin. This work was sponsored by U.S. Army ARDEC, U.S. Marine Corps, and The University of Texas at Austin.

\section{REFERENCES}

[1] M.D. Werst, et al, "Continued testing of the cannon caliber electromagnetic gun system (CCEMG)," submitted to the 9th EML Symposium, Edinburgh, Scotland, May, 1998.

[2] D.J. Wehrlen, R.A. Lee, and R.F. Thelen, "Power electronics and controls for air core compulsator," IEEE Transactions on Magnetics, Vol. 31 No. 1, Jan. 1995.

[3] C.E. Penney and T.J. Hotz, "Design and testing of the power electronics for the cannon caliber electromagnetic gun system," IEEE Transactions on Magnetics, Vol. 33 No. 1, Jan. 1997.

[4] R.A. Lee, D.J. Wehrlen and C.E. Penney, "Design and testing of a coaxial opening switch and solid state closing switch for the cannon caliber electromagnetic launcher system (CCEMG)," 10th IEEE Pulsed Power Conference, Albuquerque, NM, July 10-13, 1995. 